\title{
Effects of exercise on fiber properties in the denervated rodent diaphragm
}

\author{
Hidetaka Imagita ${ }^{1,}$, , Susumu Sakata ${ }^{1}$, Akira Minematsu ${ }^{1}$, Naohiko Kanemura ${ }^{2}$, Hideki Moriyama ${ }^{3}$, \\ Hidenori Takemoto ${ }^{4}$, Tadayuki Mita ${ }^{1,5}$
}

${ }^{1}$ Graduate School of Health Sciences, Kio University, Nara, Japan

${ }^{2}$ Department of Physical Therapy, Faculty of Health Sciences, Saitama Prefectural University, Saitama, Japan

${ }^{3}$ Department of Physical Therapy, Faculty of Health Sciences, Kobe University, Hyogo, Japan

${ }^{4}$ Department of Physical Therapy, Faculty of Health Sciences, Hiroshima Prefectural University, Hiroshima, Japan

${ }^{5}$ Department of Rehabilitation, Akiyama Orthopedic Hospital, Mie, Japan

\section{Email address:}

h.imagita@kio.ac.jp (Imagita H.), s.sakata@kio.ac.jp (Sakata S.), a.minematsu@kio.ac.jp (Minematsu A.),

kanemura-naohiko@spu.ac.jp (Kanemura N.), morihide@harbor.kobe-u.ac.jp (Moriyama H.),

takemoto@pu-hiroshima.ac.jp (Takemoto H.), mita.tadayuki@lemon.or.jp (Mita T.)

\section{To cite this article:}

Hidetaka Imagita, Susumu Sakata, Akira Minematsu, Naohiko Kanemura, Hideki Moriyama, Hidenori Takemoto, Tadayuki Mita. Effects of Exercise on Fiber Properties in the Denervated Rodent Diaphragm. American Journal of Biomedical and Life Sciences.

Vol. 2, No. 6, 2014, pp. 141-145. doi: 10.11648/j.ajbls.20140206.11

\begin{abstract}
In a unilaterally denervated diaphragm, atrophy of fast-twitch glycolytic (FG) fibers, but not of fast-twitch oxidative glycolytic (FOG) or slow-twitch oxidative (SO) fibers, occurs. The inhibition of atrophy in FOG and SO fibers may be due to the effects of stretch stimuli caused by respiratory contraction of the contralateral diaphragm. We examined the effects of exercise (treadmill running) on the histochemical and biochemical properties of muscle fibers of the costal diaphragm in three groups. Eighteen rats were distributed in (i) a denervation + treadmill running (Dn ex) group, (ii) a denervation (Dn) group, and (iii) a control (Ct) group. After 4 weeks of running, we measured the expression of myosin heavy chain (MHC) isoforms and cross-sectional area of the diaphragm muscle. The expression of MHC 1 and MHC 2a increased 4 weeks after denervation. In contrast, the expression of MHC $2 \mathrm{~d}$ decreased significantly. After 4 weeks of running, the cross-sectional area of FG fibers increased significantly $(85.5 \%$; $\mathrm{P}<0.05)$ compared with that in the Dn group. We suggest that exercise enhances the morphological properties and myosin heavy chain isoforms of the denervated diaphragm. This exercise-mediated adaptation may be associated with the improvement of FG fibers in the denervated diaphragm.
\end{abstract}

Keywords: Denervated Diaphragm Muscle, Treadmill Running, Stretching, Histochemistry, Myosin Heavy Chains Isoform

\section{Introduction}

The diaphragm is a dome-shaped skeletal muscle with peripheral muscle fibers that converge into a central tendinous chord. It plays a significant role in respiration. The characteristic action of the diaphragm muscle is highly effective synchronization of both sides, and it enables the muscle twitch and relaxation to be repeated continuously (approximately 80 times per min). The phrenic nerve is the only motor nerve innervating the diaphragm; the left and right phrenic nerves innervate the left and right side of the diaphragm, respectively.

In a previous study, G.C. Sieck, et al. reported that although fast-twitch glycolytic (FG) fibers caused atrophy in the denervated diaphragm (unilateral phrenicotomy) and that neither fast-twitch oxidative glycolytic (FOG) nor slow-twitch oxidative (SO) fibers appeared to contribute [1-3]. It is thought that unilateral phrenic nerve denervation paralyzes the ipsilateral diaphragm muscle, rendering it inactive. However, contraction of the contralateral diaphragm muscle applies intermittent stretching synchronized with respiration. Stretch is a treatment to improve physical flexibility with continuous extension of muscle fibers or soft tissues. It also enables efficient muscle tone and smooth joint action. In addition, it has been reported that the mechanical stimulation of stretch can advance the hypertrophy of muscle fibers [4].

We hypothesized that unilateral denervation of the diaphragm incurs progressive atrophy that can be minimized 
with exercise (treadmill running) for 4 weeks in rats. To test this hypothesis, we analyzed the morphological properties and myosin heavy chain (MHC) isoforms of diaphragm muscle fiber types in denervated diaphrams.

\section{Materials and Methods}

\subsection{Experimental Animals}

Twenty-one 9-week-old male Wistar rats (body weight, $352.4 \pm 32.3 \mathrm{~g}$ ) were used in this study. The temperature and humidity of the animal holding room were maintained at $23^{\circ} \mathrm{C}$ and $50 \%$, respectively, with a 12-h light/dark cycle. Throughout the rearing period, the rats had free access to food and water. This experiment was conducted with the approval of Kio University Animal Experiment Ethical Review Board and in accordance with the Kio University Animal Experiment Management Regulations.

The animals were randomly divided into 3 groups: (i) a sham control group ( $\mathrm{Ct}$ group; $\mathrm{n}=6)$, (ii) a unilaterally denervated diaphragm group (Dn group; $n=6$ ), and (iii) a unilaterally denervated diaphragm group that performed treadmill running ( $\mathrm{Dn}$ ex group; $\mathrm{n}=6$ ). Unilateral phrenicotomy was performed in the Dn groups. Three animals were used to record diaphragm muscle movement using a digital video camera before and after the denervation surgery.

\subsection{Denervation of the Diaphragm}

In order to analyze the displacement activity of the diaphragm muscle before and after cutting the phrenic nerve, 5- and $10-\mathrm{mm}$ square markers were connected to the diaphragm muscle. The length that the denervated diaphragm muscle extended between the markers was measured on the horizontal surface. After the induction of deep anesthesia by intraperitoneal administration of a mixture of ketamine (75 $\mathrm{mg} / \mathrm{kg}$ body weight) and xylazine (10 $\mathrm{mg} / \mathrm{kg}$ body weight), a medial incision measuring approximately $4 \mathrm{~cm}$ long was placed on the ventral side of the neck. The superficial muscles were carefully detached under a stereoscopic microscope, and the right phrenic nerves were exposed. The activity of the bilateral diaphragm was arrested by carefully removing $3 \mathrm{~cm}$ or more of the phrenic nerve fibers. For the $\mathrm{Ct}$ group, the same procedure was performed, except that phrenicotomy was not conducted. We carefully observed the body condition and behavior of animals for 3 days after surgery.

\subsection{Treadmill Running}

On postoperative day 3, the Dn ex group was exposed to treadmill running for $7 \mathrm{~m} / \mathrm{min}$ for a 5 -min period to become accustomed to the activity. On postoperative day 4, the Dn ex group began to run on a treadmill for $40 \mathrm{~min} /$ day at an intensity of $10-20 \mathrm{~m} / \mathrm{min}, 6$ days per week for 4 weeks. After 4 weeks of treadmill running, pentobarbital sodium salt $(50$ $\mathrm{mg} / \mathrm{kg}$ ) was inserted into the abdominal cavity of rats before anesthesia. An abdominal surgery was then performed to extract the diaphragm muscle from the denervated side.
Diaphragm specimens were frozen in liquid nitrogen and stored at $-80^{\circ} \mathrm{C}$ for future analysis.

\subsection{Morphological Characteristics of the Diaphragm}

Histochemical analysis was performed for the frozen muscle samples that exhibited an optimum length. Sequential, cross-sectional, $10-\mu \mathrm{m}$ sections were collectd at $-20^{\circ} \mathrm{C}$ using a cryostat (CM1850, Leica microsystems, Germany). The succinic dehydrogenase (SDH) complex and myosin ATPase complex were examined. Photographic images of the myosin ATPase complex and SDH complex were examined and fibers were classified into three types: SO fibers (light color), FOG fibers (dark color), and FG fibers (semi-dark color) on figure 2A. The cross-sectional area of all measurable muscle fibers was measured using image analysis software (Image J version 1.46).

\subsection{MHC Isoform Analysis}

Using the biochemical specimens, MHC was isolated by modifying the method of Doucet et al. [5] for sodium dodecyl sulfate polyacrylamide gel electrophoresis (SDS-PAGE). The muscle tissue was trimmed to $15-25 \mathrm{mg}$ and homogenized in a micrtotube, and MHC was extracted with 40 volumes of an extractant, and the extract was diluted 75 times with an incubation medium and subjected to electrophoresis. The myofibrillary protein extract prepared was injected at 5 $\mu \mathrm{L} /$ lane and elecrophoresed at $4^{\circ} \mathrm{C}$ for $48 \mathrm{~h}$ at a fixed voltage of $170 \mathrm{~V}$. The gel obtained by electrophoresis was stained with silver, and the compositional ratios of various bands (MHC1, $2 \mathrm{a}, 2 \mathrm{~b}$, and $2 \mathrm{~d}$ ) were calculated using image analysis software (Image J version 1.46).

\subsection{Statistical Analyses}

All results are presented as means \pm standard deviations. One-way ANOVA was performed to compare the cross-sectional area for each muscle fiber type among three groups, and multiple comparisons were made using Sheffe's procedure. The Mann-Whitney $U$ test was performed for MHC isoform analyses. The level of statistical significance was set at $\mathrm{P}<0.05$.

\section{Results}

\subsection{Movement of Denervated Hemidiaphragm Muscles During Respiration}

The movement of the right and left diaphragm muscles was recorded using a digital video camera before and after removing the phrenic nerve in three rats. Before phrenic nerve removal, the diaphragm muscle contracted symmetrically and lowered during inspiration.After cutting the phrenic nerve, the left diaphragm muscle became inactive, and no muscular contraction was observed. Minimal horizontal movement was observed. The contraction on the contralateral side made the left diaphragm muscle extend during inspiration. The displacement of activity in the left diaphragm muscle was 
observed toward the right diaphragm muscle on the horizontal surface. Analysis of the video images demonstrated that the denervated diaphragm muscle extended by $11.2 \pm 6.4 \%$ on the horizontal surface (Fig. 1).
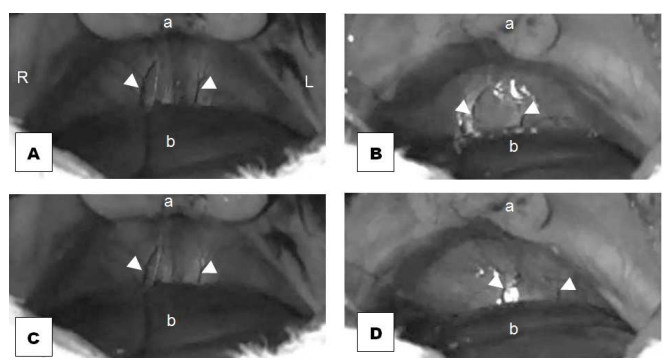

Figure 1. Photomircographs of the horizontal surface of the diaphragm from a control $(A \& C)$ and denervated animal $(B \& D)$ during the relaxation $(A \&$ $B)$ and contraction phases $(C \& D)$. a, xiphoid process; $b$, liver; arrows, inferior phrenic artery

\subsection{Morphological Characteristics of the Diaphragm Muscles}

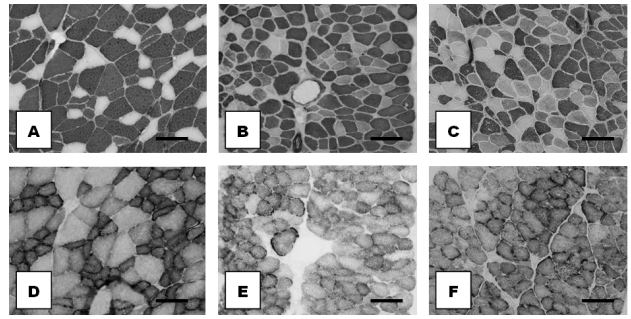

Figure 2. Photomicrographs of cross-sections through the diaphragm muscle that have been stained for adenosine triphosphatase (ATPase; $A, B$, and $C$ ) and succinate dehydrogenase ( $S D H ; D, E$, and $F$ ) from the control $(A \& D)$, denervation $(B \& E)$, and denervation and exercise $(C \& F)$ groups Scale bar $=100 \mu \mathrm{m}$

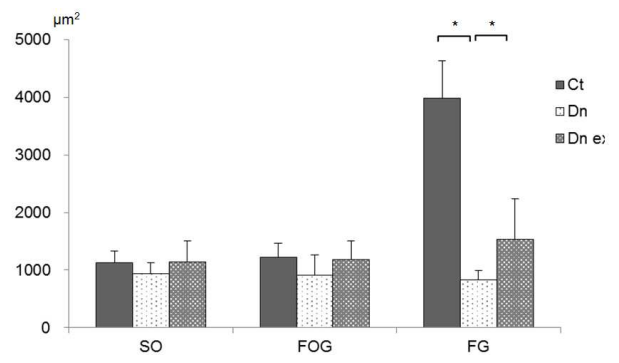

Figure 4. Cross-sectional area for each muscle fiber type in the control, denervation, and denervation + exercise groups

Investigation of the cross-sectional area (CSA) in each group according to muscle fiber type (Fig. 2) revealed that the CSA of SO fibers in the Dn group was the smallest. However, this decrease was not significant compared with that in the other groups (Ct group, $1123.8 \pm 202.0 \mu \mathrm{m}^{2}$; Dn group, 939.5 $\pm 195.5 \mu \mathrm{m}^{2}$; Dn ex group, $1141.2 \pm 372.3 \mu \mathrm{m}^{2}$ ). The CSA of FOG fibers was also smallest in the Dn group. This change was also not significant among the 3 groups (Ct group, 1222.7 $\pm 238.6 \mu \mathrm{m}^{2}$; Dn group, $913.8 \pm 351.9 \mu^{2}$; Dn ex group, $\left.1180.8 \pm 327.8 \mu \mathrm{m}^{2}\right)$. The CSA of FG fibers in the Dn and Dn ex groups decreased significantly compared with that in the $\mathrm{Ct}$ group (Ct group, $3986.1 \pm 655.1 \mu^{2}$; Dn group, $824.7 \pm$
$162.4 \mu \mathrm{m}^{2}$; Dn ex group, $1529.8 \pm 711.9 \mu \mathrm{m}^{2}$ ). Moreover, the Dn group decreased significantly compared with Dn ex group (Figure 4).

SO, slow-twitch oxidative fibers; FOG, fast-twitch oxidative glycolytic fibers; FG, fast-twitch glycolytic fibers $* \mathrm{P}<0.05$ vs the Dn group

\subsection{Composition of the MHC Isoforms}

The MHC isoform composition was analyzed using SDS-PAGE (Fig. 3, Table 1). In the $\mathrm{Ct}$ group, MHC 2d accounted for $53.3 \pm 9.2 \%$ of the total MHC isoforms. MHC2a was the second most common component $(26.0 \pm 6.7 \%)$, followed by MHC1 $(17.3 \pm 7.3 \%)$ and MHC2b $(3.5 \pm 3.4 \%)$. In the Dn group, MHC 2a accounted for $42.5 \pm 6.4 \%$ of the total isoforms, followed by MHC $1(39.2 \pm 19.0 \%)$ and MHC $2 \mathrm{~d}(18.4 \pm 15.1 \%)$; MHC2b was not detected. Compared with that in the $\mathrm{Ct}$ group, the expression of MHC $1(226.6 \%$; $\mathrm{P}<$ $0.05)$ and MHC $2 \mathrm{a}(163.5 \% ; \mathrm{P}<0.05)$ increased, whereas the relative expression of $\mathrm{MHC} 2 \mathrm{~d}(34.5 \%$; $\mathrm{P}<0.05)$ decreased.

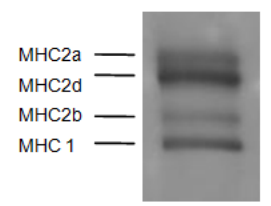

A

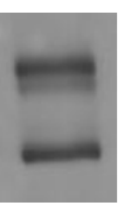

B

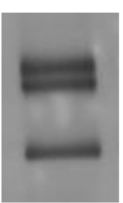

$\mathrm{C}$
Figure 3. Myosin heavy chain (MHC) isoform composition of the rat diaphragm muscle was determined by densitometric analysis of sodium dodecylsulfate polyacrylamide gel electrophoresis (SDS-PAGE). Representative gels from the control $(A)$, denervation $(B)$, and denervation + exercise (Dn ex) groups are shown.

Table 1. Percentage distribution of myosin heavy chain isoforms in diaphragm muscles from each group (Ct, Dn, and Dn ex)

\begin{tabular}{llll}
\hline & Ct & Dn & Dn ex \\
\hline MHC 1 & $17.3 \pm 7.3$ & $39.2 \pm 19.0^{*}$ & $35.0 \pm 7.5^{*}$ \\
MHC 2a & $26.0 \pm 6.7$ & $42.5 \pm 6.4^{*}$ & $39.8 \pm 7.8^{*}$ \\
MHC 2d & $53.3 \pm 9.2$ & $18.4 \pm 15.1^{*}$ & $23.0 \pm 6.1^{*}$ \\
MHC 2b & $3.5 \pm 3.4$ & $0^{*}$ & $2.2 \pm 3.1$ \\
\hline
\end{tabular}

* vs. Ct group $(\mathrm{p}<0.05)$

In the Dn ex group, MHC 2a accounted for $39.8 \pm 7.8 \%$ of the total isoforms detected, while MHC 1 accounted for $35.0 \pm$ $7.5 \%$, MHC $2 \mathrm{~d}$ for $23.0 \pm 6.1 \%$, and MHC $2 \mathrm{~b}$ for $2.2 \pm 3.1 \%$. Compared with that in the $\mathrm{Ct}$ group, the relative expression of MHC $1(202.3 \% ; \mathrm{P}<0.05)$ and MHC $2 \mathrm{a}(153.1 \% ; \mathrm{P}<0.05)$ increased, whereas that of MHC2d $(43.2 \% ; \mathrm{P}<0.05)$ decreased. However, a significant difference was not found in the comparison between the Dn group and the Dn ex group.

\section{Discussion}

The present study investigated the movement of denervated hemidiaphragm muscles during respiration, changes in the diaphragm muscle after phrenicotomy, and changes in the diaphragm muscle on performing intense physical activity in the form of treadmill runnning. 


\subsection{Movement of Denervated Hemidiaphragm Muscles During Respiration}

Consistent with a previous study performed in the rabbit, after phrenicotomy, the denervated diaphragm muscle became inactive, and muscular contractions were not observed. Contractions on the intact side resulted in the denervated side extending during inspiration, and muscle displacement (18\%) occurred in the direction of intact diaphragm muscle on the horizontal surface. The central tendon moved by approximately $4.1 \pm 0.5 \mathrm{~mm}$. In the rabbit, unilateral denervation caused a displacement of $5 \%$ extension during inspiration [6]. Respiratory contraction of the contralateral diaphragm brought about mechanical stretch to the denervated diaphragm muscle and may have produced the characteristic changes of muscular hypertrophy in the SO fibers and FOG fibers. Therefore, the denervated diaphragm muscle appears to be affected by the intact contralateral diaphragm.

\subsection{Changes in the Denervated Diaphragm Muscle}

The denervated diaphragm muscle, by unilateral phrenic nerve denervation, tended to decrease the CSA regardless of fiber type (SO fibers, 16\%; FOG fibers, $25 \%$; FG fibers, $79 \%$ ). Therefore, the FG fibers were particularly atrophied compared with the other fiber types. In terms of $\mathrm{MHC}$ isoform composition of the muscle, MHC $2 \mathrm{~d}$ and $2 \mathrm{~b}$ were significantly decreased.

In a previous study, we suggested that FG fibers in the denervated diaphragm muscle exhibit severe atrophy and appear to influence the contractile properties of the diaphragm [7]. Because the muscle protein composition of the denervated model used in this study is more remarkably controlled compared with that of other atrophy models, the denervated model exerts the greatest influence on muscular atrophy [8]. The method most commonly used to examine denervated muscle function is severing the sciatic nerve and denervating the soleus, plantar, and gastrocnemius muscles. This model also decreases the CSA of muscle fibers (Fig. 4). It has been also reported that slow-twitch fibers develop into fast-twitch fibers in the soleus muscle (slow to fast transformation) $[9,10]$. However, in this study, we observed the opposite. That is, in the Dn group, development of slow-twitch muscle fibers was observed. Geiger et al. reported that the amount of protein in all MHC isoforms in the denervated diaphragm also decreases at 1-3 days after denervation, but they observed that $\mathrm{MHC} 1$ and $2 \mathrm{a}$ isoforms were slightly increased at 3-7 days and that there were no changes after 7-14 postoperative days [11]. Moreover, mRNA expression of the MHC protein increased 1 day after denervation, whereas it decreased significantly at 3,7 , and 14 days after denervation. Geiger et al. indicated that MHC mRNA expression did not correspond to the amount of MHC protein in the denervated diaphragm [11]. This may explain the differences in the atrophy of different fibers types observed. In the denervated diaphragm, the transcription, translation, and post-translational regulation of the MHC gene may be specifically regulated by denervation. Moreover, the MHC mRNA transcription and the protein turnover ratio (protein synthesis and degradation) may be selectively controlled and therefore could influence MHC protein expression. The muscle atrophy caused by denervation prevents tension generation and changes in the length of skeletal muscle. It is thought that the intermittent stretch synchronized with respiration affects the denervated diaphragm and prevents normal muscle function.

\subsection{Effects of Treadmill Running on the Denervated Diaphragm}

In the present study, rats ran $10-20 \mathrm{~m} / \mathrm{min}$ for $40 \mathrm{~min}$, which equates to moderate-intensity exercise $[12,13]$. We suggest that this level of exercise is sufficient to inhibit a decrease in muscle fiber CSA. The FG fibers were particularly affected by treadmill running. It is possible that the contractile activity of the diaphragm increased with exercise and that the stretch to the denervated diaphragm resulted in this inhibition.

It has been reported that the disuse atrophy of type I fibers of the soleus muscle caused by arthrodesia can be reversed by a short period (1 week) of stretching. Hypertrophy of both type I and II fibers is evident after 3 weeks [14]. Fixing the stretched soleus muscle to the maximum dorsiflexion position in the hindlimb inhibits the muscle atrophy of only type II fibers $[15,16]$. Continuous stretching of denervated muscle significantly increases the diameter of type I and II fibers in the soleus muscle and type IIA and IIB fibers in the extensor digitorum longus muscle 3 weeks after sciatic nerve denervation [17]. Continuous muscle stretch inhibited muscle atrophy. Furthermore, decreases in MHC1 mRNA and MHC2a mRNA of the denervated gastrocnemius and soleus muscles were reversed by muscle stretching [18-20]. In our study, the denervated diaphragm may prevent muscle atrophy of the FG fibers via respiratory contractions originating in the contralateral intact diaphragm. The mechanical stretching may also increase with increased respiratory contractions associated with running. However, further studies are required to eludicate the effects of factors such as hormone secretion that may alter muscle proteins and the increase in blood flow to the muscle during running, which may also influence muscle atrophy caused by denervation.

There are some limitations of this study. One limitation of this study relates to the sample size. Since only 6 rats per one group were evaluated, wish be more careful in extrapolating the results of this study to human. A second limitation of this study was that although the rat is an accepted model of muscle stretch on denervated muscle because of its morphologic similarities to humans, it is not a clinical study. However, it has suggested that this type of stretch would be able to become new rehabilitation approach for prevention of atrophy in paralyzed muscle and inactive muscle. For example, if a little extension of muscular fibers can be obtained by continuous joint motion on the medical equipments such as CPM (continuous passive motion) machine, it will support on prevention of atrophy of muscle fibers. 


\section{Conclusions}

This study demonstrated that treadmill running improves both functional and morphological properties of the denervated diaphragm muscle in rats. We propose that exercise-mediated adaptation is related to the improvement of muscle atrophy as a result of muscle stretching during respiration.

\section{Acknowledgements}

This research was supported in part by a Grant-in-Aid for Scientific Research (C) from Japan Society for the Promotion of Science; 20500473 and 24500628. This research is finding based on Kio University Fellowship for Scholars and Researchers to Study Abroad.

\section{References}

[1] B. Aravamudan, C.B. Mantilla, W.Z. Zhan and G.C. Sieck, "Denervation effects on myonuclear domain size of rat diaphragm fibers,"Journal of Applied Physiology, vol.100, no.5, pp.1617-1622, 2006

[2] M.I. Lewis, T.J. Lorusso, W.Z. Zhan and G.C. Sieck, "Interactive effects of denervation and malnutrition on diaphragm structure and function,"Journal of Applied Physiology, vol.81, no.5, pp.2165-2172, 1996.

[3] W.Z. Zhan and G.C. Sieck, "Adaptations of diaphragm and medial gastrocnemius muscles to inactivity,"Journal of Applied Physiology, vol.72, no.4, pp.1445-1453, 1992.

[4] C. Rommel, S. Bodine, B. Clarke, R. Rossman, L. Nunez, T. Stitt, G. Yancopoulos and D.J. Glass, "Mediation of IGF-1-induced skeletal myotube hypertrophy by $\mathrm{PI}(3) \mathrm{K} / \mathrm{Akt} / \mathrm{mTOR}$ and $\mathrm{PI}(3) \mathrm{K} / \mathrm{Akt} / \mathrm{GSK} 3$ pathways,"Nature Cell Biology, vol.3, no.11, pp.1009-1013, 2001.

[5] J.P. Doucet and J.M. Trifaró, "A discontinuous and highly porous sodium dodecyl sulfate-polyacrylamide slab gel system of high resolution,"Anal Biochem, vol.168, no.2, pp.265-271, 1988.

[6] W.Z. Zhan, G.A. Farkas, M.A. Schroeder, L.E. Gosselin and G.C. Sieck, "Regional adaptations of rabbit diaphragm muscle fibers to unilateral denervation,"Journal of Applied Physiology, vol.79, no.3, pp.941-950, 1995.

[7] H. Imagita, Y. Saeki, E. Shono, O. Yoshimura, Y. Tobimatsu and H. Miyata, "The effect of aging on functional and morphological changes in the denervated diaphragm muscle of rats,"Journal of the Japanese Physical Therapy, vol.33, no.1, pp.22-29, 2006.

[8] T. Morinaga (editing), "Exercise and Physiological Functions,"NAP limited, pp.108-122, 1999. (In Japanese)
[9] A. d'Albis, R. Couteaux, F. Goubel, C. Janmot and J.C. Mira, "Response to denervation of rabbit soleus and gastrocnemius muscles. Time-course study of postnatal changes in myosin isoforms, fiber types, and contractile properties,"Biology of the Cell, vol.85, no.1, pp.9-20, 1995.

[10] K.A. Huey and S.C. Bodine, "Changes in myosin mRNA and protein expression in denervated rat soleus and tibialis anterior,"European Journal of Biochemistry, vol.256, no.1, pp.45-50, 1998.

[11] P.C. Geiger, J.P. Bailey, W.Z. Zhan, C.B. Mantilla and G.C. Sieck, "Denervation-induced changes in myosin heavy chain expression in the rat diaphragm muscle,"Journal of Applied Physiology, vol.95, no.2, pp.611-619, 2003.

[12] T. Saito and H. Soya, "Changes of hypothalamic ACTH secretagogue (CRH/AVP) during running stress in rats,"Journal of Exercise and Sports Physiology, vol.12, no.1, pp.33-39, 2005. (In Japanese)

[13] T. Saito and H. Soya, "Delineation of responsive AVP-containing neurons to running stress in the hypothalamus,"American Journal of Physiology Regulatory, Integrative and Comparative Physiology, vol.286, no.3, pp.R484-R490, 2004.

[14] M. Okita, T. Yoshimura, J. Nakano, A. Saeki, A.Uehara, A. Mineshita and K. Eguchi, "Effects of Short Duration Stretching on Disuse Muscle Atrophy in Immobilized Rat Soleus Muscles,"Journal of the Japanese Physical Therapy Association, vol.4, no.1, pp. 1-5, 2001.

[15] T. Yamazaki, "Influence of Hindlimb Unweighting and Intermittent Weight Bearing on Dynamics of Nuclei in Rat Soleus Muscle,"Journal of the Japanese Physical Therapy Association, vol.6, no.1, pp. 1-8, 2003.

[16] N. Agata, N. Sasai, M. Inoue-Miyazu, K. Kawakami, K. Hayakawa, K. Kobayashi and M. Sokabe, "Repetitive stretch suppresses denervation-induced atrophy of soleus muscle in rats,"Muscle \& Nerve, vol.39, no.4, pp.456-462, 2009.

[17] S.Kimura, T. Inaoka, T. Yamazaki, "Influence of Passive Stretching on Inhibition of Disuse Atrophy and Hemodynamics of Rat Soleus Muscle,"Journal of the Japanese Physical Therapy Association, vol.15, no.1, pp. 9-14, 2013.

[18] P.T. Loughna and M.J. Morgan, "Passive stretch modulates denervation induced alterations in skeletal muscle myosin heavy chain mRNA levels,"Pflügers Archiv, vol.439, no.1, pp.52-55, 1999.

[19] D.G. Allen, G.D. Lamb and H. Westerblad, "Skeletal muscle fatigue: cellular mechanisms,"Physiological reviews, vol.88, no. 1 , pp.287-332, 2008 .

[20] H.M. Argadine, N.J. Hellyer, C.B. Mantilla, W.Z. Zhan and G.C. Sieck, "The effect of denervation on protein synthesis and degradation in adult rat diaphragm muscle,"Journal of Applied Physiology, vol.107, no.2, pp.438-444, 2009. 